\title{
Rendimiento de plantas de tomate injertadas y efecto de la densidad de tallos en el sistema hidropónico
}

\author{
Roberta M.N. Peili' ${ }^{1}$ José L. Gálvez ${ }^{2}$ \\ ${ }^{1}$ UFPel, FAEM, Dept ${ }^{\circ}$ Fitotecnia, C. Postal 354, 960010-900 Pelotas-RS; Becaria de Doctorado del CNPq; ${ }^{2} \mathrm{UAL}_{\text {, }}$ Dept ${ }^{\circ}$ Economía, \\ Sociología y Política Agrarías; 04120 Almería, España; E-mail: rmpeil@ufpel.tche.br
}

\section{RESUMEN}

El cultivo de tomate en primavera con baja densidad inicial de plantas y luego aumentar-se la densidad efectiva mediante el desarrollo de tallos laterales, cuando las integrales diarias de radiación son altas, parece ser una manera prometedora de incrementar el número de frutos $\mathrm{m}^{-2} \mathrm{y}$ uniformizar el tamaño del fruto; y presenta la ventaja adicional de requerir-se un menor número de plantas para la superficie de cultivo. Por lo tanto, se ha llevado a cabo un ensayo cuyo objetivo ha sido evaluar los efectos de la densidad de tallos sobre el rendimiento y la uniformidad de los frutos de un cultivo de tomate injertado y no injertado. El cultivo se realizó de marzo a julio de 1998, en las condiciones de invernadero en Almería (sudeste español). La densidad inicial de plantación fue de 2,4 y 3,0 plantas $\mathrm{m}^{-2}$, en el caso de las plantas no injertadas, que presentaban un único tallo en el transplante; y 1,2 plantas $\mathrm{m}^{-2}$ en las plantas injertadas, que tenían dos tallos $\left(2,4\right.$ tallos $\left.\mathrm{m}^{-2}\right)$. A partir del mes de abril, mediante el desarrollo de tallos laterales (cuando el tratamiento así lo exigía) se pasó a tener las siguientes densidades: 2,4; 3,0 y 3,5 tallos $\mathrm{m}^{-2}$ (plantas injertadas y no injertadas); y 3,0 plantas no injertadas $\mathrm{m}^{-2} \mathrm{a}$ un tallo durante todo el ciclo. Los resultados muestran que plantas de tomate injertadas y transplantadas a dos tallos tuvieron igual producción total, pero frutos de tamaño más uniforme, que plantas no injertadas transplantadas a un tallo y con doble densidad de plantas. Dentro del rango de 2,4 a 3,5 tallos $\mathrm{m}^{-2}$, una mayor densidad de tallos aumentó la producción precoz y dio un tamaño más uniforme de frutos, no afectando su peso promedio y la producción total. La adición de tallos laterales $\left(3,0\right.$ tallos $\left.\mathrm{m}^{-2}\right)$ supuso una reducción de un $20 \%$ en el rendimiento total, pero vino acompañada por un ahorro de un $21 \%$ y de un $39 \%$ en el número de plantas no injertadas e injertadas, respectivamente, necesarias para la misma superficie; asimismo, dio un tamaño más uniforme de fruto, comparando con plantas cultivadas desde el inicio del ciclo en alta densidad $(3,0$ plantas $\mathrm{m}^{-2}$ sin adición de tallos laterales).

Palabras-clave: Lycopersicon esculentum, invernadero, cultivo sin suelo, técnica de la lámina de nutrientes.

\author{
ABSTRACT \\ Yield of tomato crop as a result of grafting and shoot density \\ in hydroponic system
}

Tomato cultivated in spring at a low plant density and then increasing the effective plant density by means of allowing side shoots to develop, when daily light integrals are high, appears a promising way of increasing spring-season tomato crop fruit number per square meter and to produce fruits of more uniform size; and has the added advantage that fewer plants have to be purchased per cropped area. The effects of side shoots on fruit uniformity and yield of tomato crop were evaluated in a trial carried out from March to July 1998 in a greenhouse in Almería (south-eastern Spain). The initial plant densities were 2.4 and 3.0 plants $\mathrm{m}^{-2}$, in the case of non-grafted plants, which had one main stem; and 1.2 plants $\mathrm{m}^{-2}$ in grafted plants, which had two main stems $\left(2.4\right.$ shoots $\left.\mathrm{m}^{-2}\right)$ at setting. From April, by allowing side shoots to develop (when the treatment required), population densities of $2.4 ; 3.0$ and 3.5 shoots $\mathrm{m}^{-2}$ (grafted and nongrafted plants) were raised; and 3.0 non-grafted plants $\mathrm{m}^{-2}$ without side shoots during all cropping-season treatment was maintained. The results showed that grafted tomato plants set with two main stems presented the same final yield but produced fruits of more uniform size than non-grafted plants set with one main stem and double initial plant density. In a range from 2.4 to 3.5 shoots $\mathrm{m}^{-2}$, increasing shoot density increased early yield and produced fruits of more uniform size but presented no effect on the mean fruit weight and the final yield. The addition of side shoots $\left(3.0\right.$ shoots $\left.\mathrm{m}^{-2}\right)$ was accompanied by a $20 \%$ loss of final yield but from $21 \%$ and $39 \%$ fewer non-grafted and grafted plants, respectively, and produced fruits of more uniform size, relative to the high initial density treatment (3.0 plants $\mathrm{m}^{-2}$ without side shoots).

Keywords: Lycopersicon esculentum, greenhouse, soilless cultivation, nutrient film technique.

\section{(Recebido para publicação em 9 de setembro de 2002 e aceito em 4 de julho de 2003)}

$\mathrm{E}$ 1 rendimiento económico del cultivo del tomate es resultante de la combinación entre el número de frutos cosechados por unidad de área y sus tamaños individuales. El tamaño del fruto es un factor de calidad sumamente importante y debe ser lo más uniforme posible durante todo el ciclo de producción. En principio, sería posible mantener el tamaño de los frutos dentro del rango preferido por el mercado durante todo el ciclo de cultivo, si el número de frutos es alterado para coincidir con los cambios en el aporte de asimilados que acompañan los cambios de la radiación solar incidente. De un lado, esto puede ser obtenido por el pinzamiento de la inflorescencia, con reducción del número de frutos por racimo cuando las integrales diarias de radiación son bajas $\mathrm{y}$, de esta manera, incrementase el aporte de asimilados a los frutos que se quedan (Bertin e Gary, 1992). Por otro lado, se puede incrementar la densidad de plantación, al aumentar-se el número de frutos por unidad de superficie de cultivo en las épocas del año en que las integrales diarias de radiación son altas. El incremento en la densidad de plantación puede ser obtenido tanto por un mayor número de plantas por $\mathrm{m}^{2}$, como dejándose que tallos laterales se 
desarróllen para aumentar el número de tallos por metro cuadrado (Cockshull e Ho, 1995; Nederhoff et al., 1992).

El cultivo de tomate en primavera, con baja densidad inicial de plantas y luego aumentar-se la densidad efectiva mediante el desarrollo de tallos laterales, parece ser una manera prometedora de incrementar el número de frutos por metro cuadrado y presenta la ventaja adicional de tener que adquirir menor número de plantas para la superficie de cultivo. Datos tomados de investigación anterior indican que, en general, la adición de tallos laterales disminuye el peso promedio de los frutos, reduce la variabilidad del peso promedio de los frutos, que resultan ser de tamaño más uniforme durante el ciclo de cultivo, y reduce la producción de frutos de mayor tamaño. Estos resultados vienen acompañados por una disminución del rendimiento de un $5 \%$, pero con un $33 \%$ de ahorro en el número de plantas necesarias, cuando comparando con los datos obtenidos en el tratamiento de alta densidad de plantas (Cockshull e Ho, 1995).

En las condiciones de primaveraverano de la zona mediterránea, la reducción de la fotosíntesis por exceso de radiación tiene un gran peso, ya que aquí, se alcanzan valores muy altos de luminosidad lo que limita la producción de tomate en invernadero en esta época del año, debido a las altas temperaturas que se alcanzan en esta situación. Adecuar el número de tallos a las condiciones de radiación va a incrementar el sombreo y la transpiración del dosel vegetal, disminuyendo la temperatura del interior del invernadero, como lo propuesto por Stanghellini (1987), posibilitando mejorar las condiciones del cultivo del tomate en épocas calurosas, con la consiguiente incidencia en la cantidad y calidad de la cosecha.

La técnica del injerto se viene adoptando en el sur de España, sobre todo como método de lucha contra patógenos del suelo. No obstante, el empleo de un patrón vigoroso, además de dar resistencia a determinado patógeno, hace con que la planta injertada también sea vigorosa y permitiría utilizar menor densidad de plantación sin disminuir la producción. De manera tal que, cuando el factor mano-de-obra no es limitante, el uso de plantas injertadas reduciría, asimismo, los costes de implantación, además de permitir el desarrollo de plantaciones más tempranas, ya que la raíz se desarrolla con temperaturas del medio radicular relativamente menores (González, 1999), lo que podría resultar en una mayor precocidad de la cosecha, factor importante en la obtención de mejores precios en la comercialización. La introducción de resistencia a las bajas temperaturas del suelo en el cultivo de invierno de pepino (Peil et al., 1998) y en cultivo forzado de sandía, de resistencia a la seca en el cultivo de la calabaza, de resistencia al exceso de humedad y de características que incrementen la capacidad de absorción de nutrientes en el cultivo de la sandía son, asimismo, objetivos buscados con la técnica del injerto (Nawashiro, 1994).

Por lo expuesto, se ha llevado a cabo un ensayo cuyo objetivo ha sido evaluar los efectos de la densidad de tallos de plantas injertadas y no injertadas sobre los componentes del rendimiento (número y tamaño medio de los frutos) y la uniformidad del tamaño de los frutos de la cosecha del tomate cultivado en ciclo de invierno-primavera.

\section{MATERIAL Y MÉTODOS}

El trabajo se ha realizado en el invierno/primavera 1997/98, en las instalaciones que posee Dunia Export, conjuntamente con la Universidad de Almería, dentro del marco del programa de investigación CDTI/970068 del Ministerio de Industria y Energía del Gobierno Español, en Almería, sudeste de España, a $36^{\circ} 42^{\prime} \mathrm{N}$ y $2^{\circ} 47^{\prime} \mathrm{W}$.

El cultivo elegido fue el de tomate cv. Radja, y el patrón de injerto, el tomate cv. Beaufort. Las plantas fueron producidas en semillero industrial dotado de ventanas automatizadas, malla de sombreo con un $30 \%$ paso de luz y calefacción de suelo radiante. El suelo es de hormigón formando balsetas en cada nave. El sistema de riego es por inundación. Las semillas de ambas cultivares (injerto y patrón) fueron sembradas en bandejas de poliexpan (128 alvéolos) conteniendo turba, siendo injertadas (en caso de que el tratamiento así lo exija) y repicadas al taco de lana de roca de 10x10x7,5 cm, a los 36 días (injerto) y a los 59 días (patrón) tras la siembra. Las diferencias de desarrollo que hay entre cultivar y patrón y, asimismo, las que acarrea la práctica del injertado, implicaron en la necesidad de distintas fechas de siembra y repicado. El método de injertado utilizado fue el de aproximación (González, 1999). En este caso, el repicado al taco era realizado inmediatamente después de esta operación.

El transplante al invernadero fue realizado el 9/03/98, manteniéndose la plantación hasta el 2/07/98. Se dispuso de un invernadero industrial multi-túnel, de cubierta a dos aguas simétricas, eje longitudinal norte-sur, con una superficie total de $1.500 \mathrm{~m}^{2}$ y una superficie de cultivo de $1.386 \mathrm{~m}^{2}$, y como material de cerramiento se ha utilizado película de polietileno térmico de $200 \mu \mathrm{m}$ de espesor. El invernadero dispone, para el control del microclima, de mallas de sombreo de un paso de luz de $50 \%$, accionadas siempre que la radiación global exterior alcanzaba 450 $\mathrm{W} \mathrm{\textrm {m } ^ { - 2 }}$ y de un sistema de calefacción de agua caliente por tuberías de hierro que impedía que la temperatura nocturna bajara de $15^{\circ} \mathrm{C}$. Estos equipos iban conectados a un ordenador que controlaba la apertura y cierre de ventanas, el plegado y desplegado de las mallas de sombreo, el arranque y la parada del sistema de calefacción, de acuerdo con parámetros predeterminados para el cultivo de tomate.

Los tratamientos experimentales fueron: $\mathrm{T} 1=$ Planta no injertada con 2,4 $(2,35)$ tallos $\mathrm{m}^{-2}$ durante todo el ciclo; $\mathrm{T} 2=$ Planta no injertada con $3,0(2,93)$ tallos $\mathrm{m}^{-2}$ a partir del mes de abril, y así hasta el final del ciclo; T3=Planta no injertada con 3,5 $(3,52)$ tallos $\mathrm{m}^{-2}$ a partir del mes de abril, y así hasta el final del ciclo; T4=Planta no injertada con 3,0 $(3,04)$ plantas o tallos $\mathrm{m}^{-2}$ durante todo el ciclo (plantas sin adición de tallos laterales);T5=Planta injertada con 2,4 $(2,35)$ tallos $\mathrm{m}^{-2}$ durante todo el ciclo; T6=Planta injertada con 3,0 (2,93) tallos $\mathrm{m}^{-2}$ a partir del mes de abril, y así hasta el final del ciclo; T7=Planta injertada 
Tabla 1. Efecto del injerto, del incremento en la densidad de tallos y de la densidad de plantación sobre la cosecha total y precoz del tomate. Almería, UAL, 1998.

\begin{tabular}{|c|c|c|c|c|c|c|}
\hline & \multicolumn{3}{|c|}{ Cosecha Total } & \multicolumn{3}{|c|}{ Cosecha Precoz } \\
\hline & $\begin{array}{c}\text { Producción } \\
\qquad\left(\mathrm{g} \mathrm{m}^{-2}\right)\end{array}$ & $\begin{array}{c}\text { No. frutos } \\
\mathrm{m}^{-2}\end{array}$ & $\begin{array}{l}\text { Peso medio del } \\
\text { fruto }(\mathrm{g})\end{array}$ & $\begin{array}{l}\text { Producción } \\
\qquad\left(\mathrm{g} \mathrm{m}^{-2}\right)\end{array}$ & $\begin{array}{c}\text { No. frutos } \\
\mathrm{m}^{-2}\end{array}$ & $\begin{array}{l}\text { Peso medio } \\
\text { del fruto (g) }\end{array}$ \\
\hline \multicolumn{7}{|c|}{ Efecto del injerto } \\
\hline No injertadas & 15580 & 97,3 & $159,8(9,1)^{2}$ & 4100 & 25,4 & 160,5 \\
\hline Injertadas & 14417 & 91,9 & $154,2(4,9)$ & 2091 & 13,3 & 156,2 \\
\hline LSD1 $(P=0,05)$ & 2227 & 11,7 & 6,4 & 554 & 4,1 & 9,9 \\
\hline \multicolumn{7}{|c|}{ Efecto del $n^{\circ}$ de tallos $m^{-2}$} \\
\hline 2,4 & 14340 & 92,0 & $155,3(8,7)$ & 2669 & 17,5 & 153,9 \\
\hline 3,0 & 15511 & 98,5 & $153,1(5,2)$ & 2962 & 19,0 & 155,1 \\
\hline 3,5 & 15518 & 93,3 & $162,6(5,9)$ & 3657 & 21,7 & 166,2 \\
\hline LSD $^{1}(P=0,05)$ & 2727 & 14,3 & 7,9 & 679 & 5,0 & 12,1 \\
\hline \multicolumn{7}{|c|}{ Efecto de la densidad de plantación } \\
\hline 3,0 plantas $\mathrm{m}^{-2}$ & 19360 & 121,7 & $159,4(5,9)^{2}$ & 4286 & 29,0 & 148,0 \\
\hline 3,0 tallos $\mathrm{m}^{-2}$ & 15511 & 98,5 & $153,1(2,1)$ & 2962 & 19,0 & 155,1 \\
\hline $\operatorname{LSD}^{1}(P=0,05)$ & 2074 & 11,1 & 19,7 & 988 & 8,2 & 37,0 \\
\hline
\end{tabular}

${ }^{1}$ LSD: Diferencia mínima significativa entre las medias de los tratamientos de injerto, entre las medias de los tratamientos del número de tallos $\mathrm{m}^{-2} \mathrm{y}$ entre las medias de los tratamientos de densidad de plantación, calculada según el teste $\mathrm{t}$ de Student.

${ }^{2}$ Entre paréntesis figuran los coeficientes de variación (\%).

con 3,5 $(3,52)$ tallos $\mathrm{m}^{-2}$ a partir del mes de abril, y así hasta el final del ciclo.

En cuanto al manejo general, partimos de una densidad inicial de 2,4 tallos $\mathrm{m}^{-2}$, excepto en el tratamiento $\mathrm{T} 4$ que fue de 3,0 tallos $\mathrm{m}^{-2}$. Las plantas injertadas tenían dos tallos iniciales (plantas a 0,90 m en la línea) y las no injertadas un tallo (plantas a 0,45 $\mathrm{m}$ en la línea, en los tratamientos T1, T2 y T3; y a $0,35 \mathrm{~m}$ en el T4). Esas condiciones conllevaron a densidades de plantación de 2,4 plantas $\mathrm{m}^{-2}$ y 1,2 plantas $\mathrm{m}^{-2}$, en los tratamientos de plantas no injertadas e injertadas, respectivamente; excepto en el tratamiento T4, en que la densidad era de 3,0 plantas $\mathrm{m}^{-2}$. En la fecha del transplante, las plantas presentaban alrededor de 12 hojas verdaderas, el primer racimo en estadio de $100 \%$ de floración y el segundo racimo en 50\% de floración. A fin de favorecer el desarrollo radical y vegetativo, se quitó la primera inflorescencia de todas las plantas. Los tallos adicionales se han dejado desarrollar inmediatamente arriba de la $5^{\mathrm{a}}$ inflorescencia. Los demás tallos laterales se han destallado sistemáticamente. El deshojado se practicó de forma sistemática, quitándose todas las hojas situadas abajo del racimo recolectado, favoreciéndose una mejor ventilación en la base de las plantas. La recolección de frutos empezó a los 54 días tras el transplante. Las plantas fueron despuntadas al alcanzar unos 2,8 m de longitud.

El cultivo se realizó mediante la técnica de NFT (del inglés Nutrient Film Technique o técnica de la lámina de nutrientes; Cooper, 1973) en canales de cultivo de polietileno flexible, blanconegro, de $200 \mu \mathrm{m}$ de espesor, longitud de $9,0 \mathrm{~m}$, anchura de $0,30 \mathrm{~m}$, pendiente de $2 \%$. La altura de la lámina de agua fue de aproximadamente $5 \mathrm{~mm}$, con una velocidad de circulación de $3 \mathrm{~L} \mathrm{~min}^{-1}$. La disposición de los canales fue en líneas dobles con distancia interna de $0,55 \mathrm{~m}$ y un pasillo de $1,33 \mathrm{~m}$ de anchura. Se disponía de un sistema automático conectado a un ordenador, para el control de la solución, del pH y de la conductividad eléctrica (CE). Dos composiciones de soluciones nutritivas ideales se han empleado durante el ciclo de cultivo. La solución inicial (utilizada en las dos primeras semanas tras el transplante) presentaba la siguiente composición (expresada en mmol L-1):
12,8 de $\mathrm{NO}_{3}^{-}, 0,5$ de $\mathrm{NH}_{4}^{+}, 1,5$ de $\mathrm{H}_{2} \mathrm{PO}_{4}^{-}$, 9,5 de $\mathrm{K}^{+}, 5,6 \mathrm{de} \mathrm{Ca}^{+2}, 2,1 \mathrm{de} \mathrm{Mg}^{+2}$ y 4,3 de $\mathrm{SO}_{4}^{-2}$; una relación $\mathrm{N} / \mathrm{K}$ de 1/2,0; y CE de $2,5 \mathrm{dSm}^{-1}$; y la solución de fructificación (a partir de la tercera semana): 13,8 de $\mathrm{NO}_{3}^{-}, 0,5$ de $\mathrm{NH}_{4}^{+}, 1,5$ de $\mathrm{H}_{2} \mathrm{PO}_{4}^{-}, 12,8$ de $\mathrm{K}^{+}, 6,1 \mathrm{de} \mathrm{Ca}^{+2}, 2,2$ de $\mathrm{Mg}^{+2}$ y 6,0 de $\mathrm{SO}_{4}^{-2}$; una relación N/ $\mathrm{K}$ de $1 / 2,5$; y CE de $3,0 \mathrm{dSm}^{-1}$.

El diseño estadístico fue en bloques al azar, con 3 repeticiones por tratamiento. A cada parcela experimental correspondió 4 líneas dobles de canales de cultivo, despreciándose las líneas bordes para las evaluaciones. Para el control de la producción (kg y número de frutos en cada pasada de recolección), cada repetición contó con 4 plantas. A partir de los datos de producción comercial de frutos, se calcularon los valores de producción precoz y total y de peso promedio de fruto en tres períodos de cosecha (precoz, media y tardía). La cosecha precoz fue determinada a través de la curva de producción acumulada del ensayo y considerada como aquella obtenida hasta la fecha que coincidía con el primer cambio significativo de la pendiente; la cosecha media 


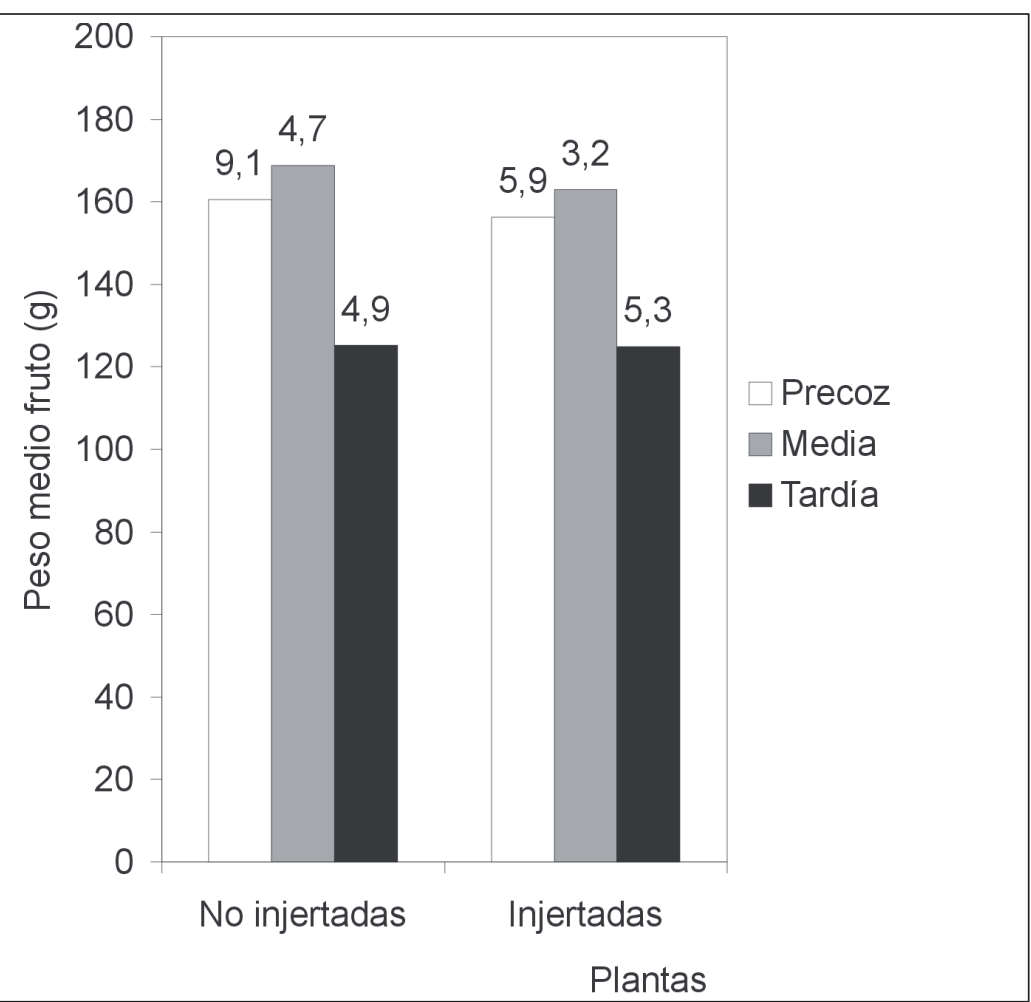

Figura 1. Peso medio del fruto en los tres períodos de cosecha (precoz, media y tardía) de plantas injertadas y no injertadas de tomate. Nota: valores sobre columnas indican los coeficientes de variación (\%). Almería, UAL, 1998.

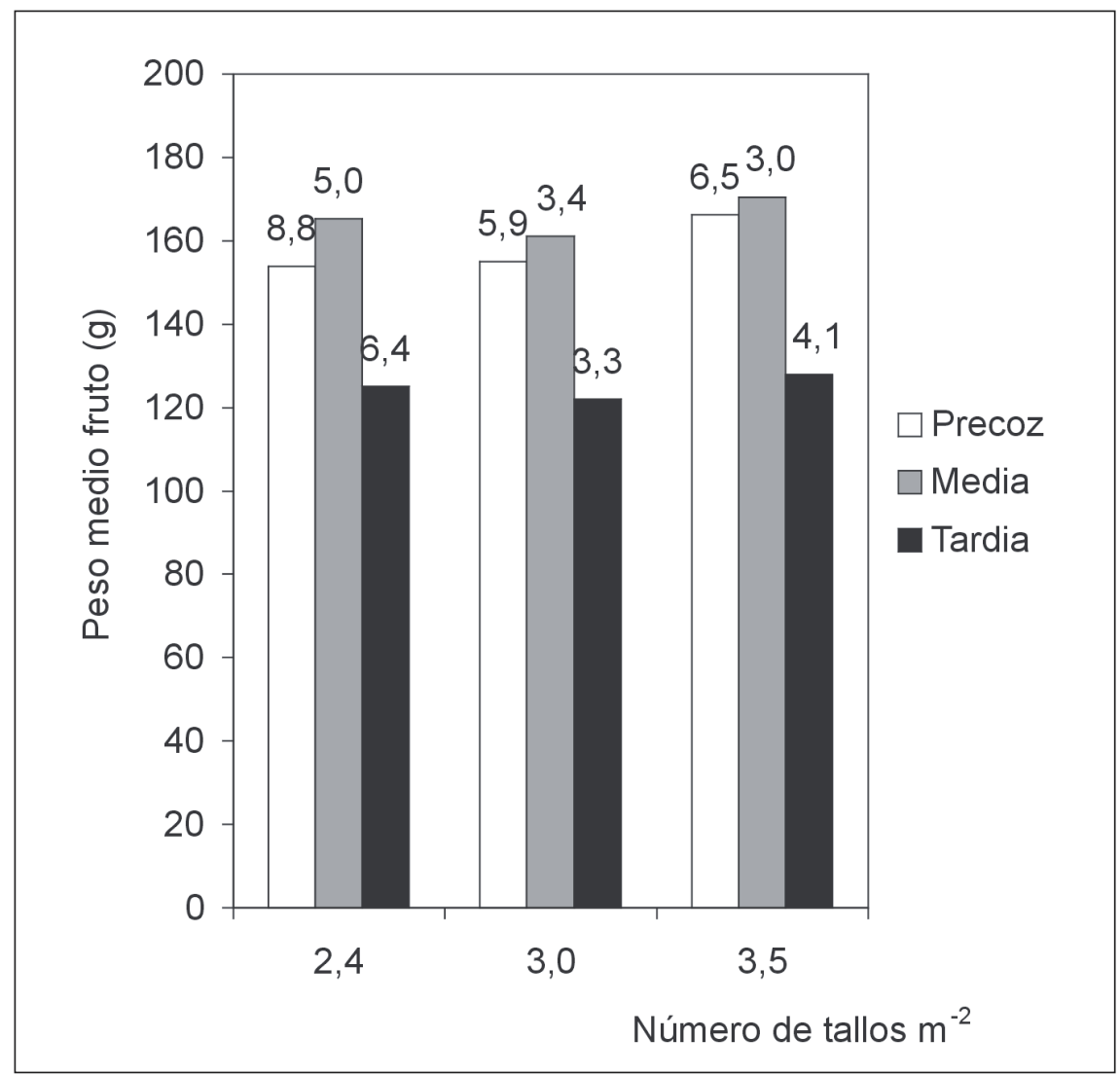

Figura 2. Peso medio del fruto en los tres períodos de cosecha (precoz, media y tardía) de los distintos niveles de densidad de tallos. Nota: valores sobre columnas indican los coeficientes de variación (\%).Almería, UAL, 1998. correspondió a la obtenida entre la cosecha precoz y la fecha que coincidía con el segundo cambio significativo de la pendiente y la cosecha tardía consideró la producción obtenida a partir de este punto. La variación del peso promedio de los frutos se ha determinado a través del coeficiente de variación de la variable.

\section{RESULTADOS Y DISCUSIÓN}

El hecho de que los análisis estadísticos indicaran que no hubo interacción significativa entre los factores injerto e incremento de la densidad de tallos, permitió estudiar los efectos de estos dos factores separadamente.

Los datos expuestos en la Tabla 1 indican que los tratamientos de injerto $\mathrm{y}$ de densidad de tallos tuvieron efecto básicamente sobre la cosecha precoz, no teniendo efecto significativo sobre la cosecha total. Las plantas no injertadas presentaron un comportamiento del componente del rendimiento de la cosecha precoz, número de frutos, superior a las plantas injertadas, que resultó en mayor producción precoz. De aquí, se puede suponer que la técnica de injerto empleada en este ensayo ocasionó un retraso en el desarrollo inicial de la planta, el cual repercutió sobre el cuajado y crecimiento de los primeros racimos. Sin embargo, estos resultados no persistieron, existiendo igualdad de los dos tratamientos en la cosecha total.

De igual manera, la mayor densidad de tallos aumentó el rendimiento de la cosecha precoz, pero, aquí, el componente significativamente afectado ha sido el peso promedio de fruto (Tabla 1). Hay que tener en cuenta que, a pesar de que las diferencias no hayan sido significativas para el número de frutos, los resultados en su conjunto indican que esta variable también tuvo un efecto determinante para la más alta producción precoz obtenida por el tratamiento de 3,5 tallos $\mathrm{m}^{-2}$.

La variación del peso promedio de los frutos (dada por el coeficiente de variación) a lo largo de las cosechas media y tardía, fue similar en plantas injertadas y plantas no injertadas (Figura 1). Sin 
embargo, las plantas injertadas produjeron frutos de tamaño más uniforme en la cosecha precoz (Figura 1) y en la cosecha total (Tabla 1) que las plantas no injertadas.

Los tratamientos de 3,0 y 3,5 tallos $\mathrm{m}^{-2}$, resultaron tener en menor variación del peso promedio del fruto en relación al tratamiento de baja densidad de tallos a lo largo de los tres períodos de cosecha (Figura 2). Esta menor variación se debe al hecho de que una mayor densidad de tallos regula la competencia por asimilados y da un tamaño más uniforme de frutos, coincidiendo, en este aspecto, con las indicaciones de distintos autores (Papadopoulos e Ormrod, 1990; Cockshull e Ho, 1995; Sánchez, 1999). Sin embargo, trabajos anteriores (Papadopoulos e Ormrod, 1990; Cockshull e Ho, 1995; Papadopoulos e Pararajasingham, 1997) muestran una reducción de la producción de los frutos de mayor tamaño, mayor número de frutos y mayor rendimiento total por $\mathrm{m}^{2}$ con el incremento de la densidad de tallos, este último efecto observado, en este ensayo, solamente en la cosecha precoz (Tabla 1). En las condiciones de primavera de la zona mediterránea la escasez de radiación solar no es un factor limitante para el cuajado y desarrollo de los frutos, lo que conllevó a que las distintas densidades de tallos presentaran igualdad de rendimiento total, coincidiendo con los resultados obtenidos por Sánchez (1999) en Almería.

En la Tabla 1 se refleja que un incremento en la densidad de tallos en plantas que a principio del ciclo tenían una densidad más baja de plantación (2,4 y 1,2 plantas $\mathrm{m}^{-2}$ en el tratamiento de plantas no injertadas e injertadas, respectivamente) pasando a 3 tallos $\mathrm{m}$ ${ }^{2}$, no logró igualar el rendimiento, tanto de la cosecha precoz, como de la cosecha total, obtenido por plantas cultivadas desde el inicio del ciclo en alta densidad de plantación (3 plantas $\mathrm{m}^{-2}$ ). Estos resultados son similares a los obtenidos por Cockshull e Ho (1995). Esta mayor producción por unidad de superficie se debe al componente de rendimiento del número de frutos que fue significativamente superior en la densidad de 3 plantas $\mathrm{m}^{-2}$ para ambas

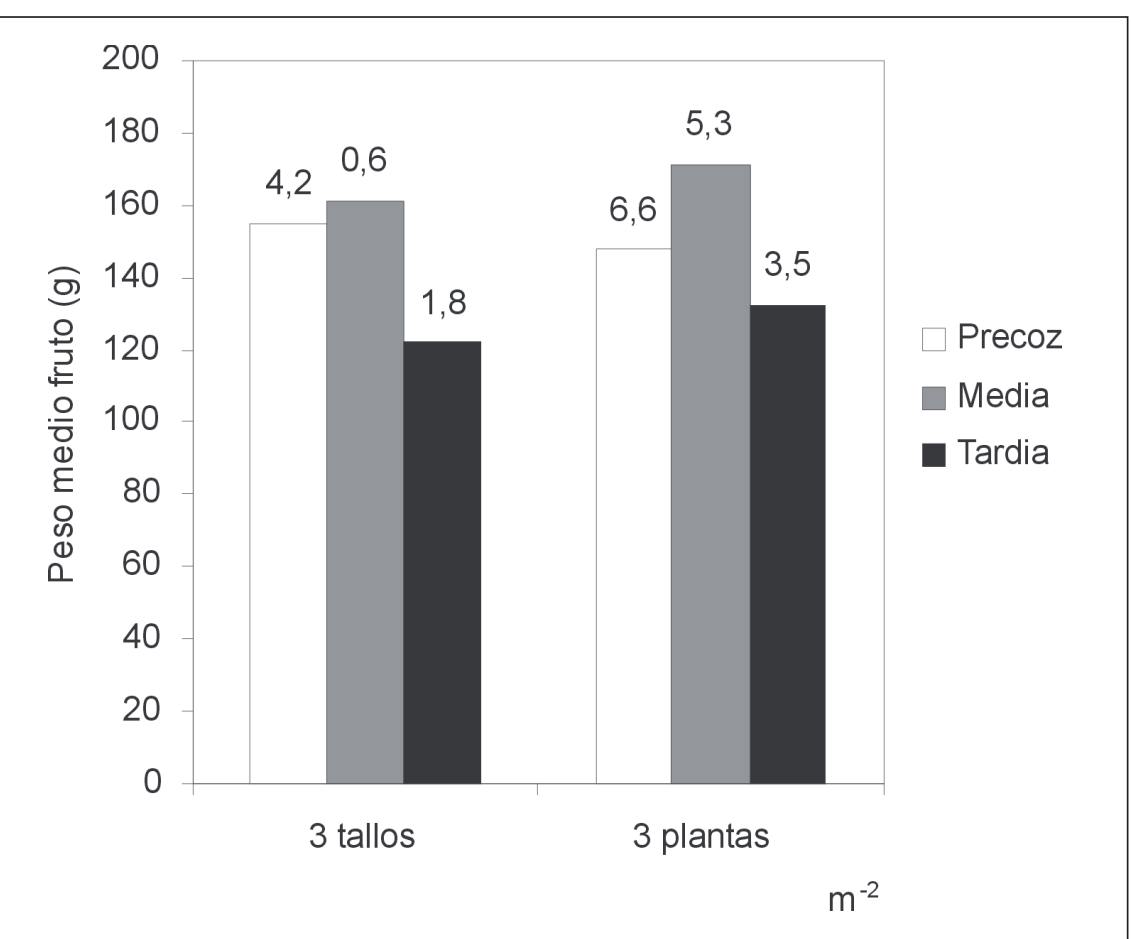

Figura 3. Peso medio del fruto en los tres períodos de cosecha (precoz, media y tardía) de plantas de tomate a una densidad de 3 plantas $\mathrm{m}^{-2}$ y de 3 tallos $\mathrm{m}^{-2}$. Nota: valores sobre columnas indican los coeficientes de variación (\%).Almería, UAL, 1998.

cosechas. Una vez que se dejaron tallos laterales a partir del quinto racimo (segundo mes tras el transplante) y que la cosecha oriunda de tales tallos empezó bastante más tarde que la cosecha de los tallos principales, se explica este menor número de frutos. Asimismo, la reducción del rendimiento, probablemente, represente una distribución de los asimilados producidos en el tallo principal hacia el tallo lateral durante su fase de desarrollo inicial. Durante dicha fase, el tallo lateral presenta una gran fuerza de sumidero y compite fuertemente por los asimilados disponibles con los frutos en desarrollo en el tallo principal, como indicado por Cockshull e Ho (1995).

Los datos obtenidos de peso promedio del fruto a lo largo del ciclo, indican que la adición de tallos laterales ocasiona una menor variación de esta variable (Figura 3 ), comparando con los datos de plantas cultivadas desde el inicio del ciclo en alta densidad y sin adición de tallos (3,0 plantas $\left.\mathrm{m}^{-2}\right)$. De un lado, esta menor variación es debida a la producción de frutos ligeramente mayores en el período de cosecha precoz, cuando el aporte de luz era menor y todavía no se habían establecido los tallos laterales, favoreciendo un crecimiento más uniforme de los frutos en el tratamiento con menor densidad de plantas. Por otro lado, cuando el aporte de luz era mayor, a partir del momento en que se establecieron los tallos laterales (cuando el cultivo ya se encontraba a mediados de primavera), se incrementó la cantidad de frutos compitiendo por asimilados, resultando en frutos de menor tamaño, pero más uniformes, durante los períodos de cosecha media y tardía.

Sobre la base de lo expuesto, hay que considerar que la adición de tallos laterales supuso una reducción en un $20 \%$ del rendimiento total, pero un ahorro de un $21 \%$ y de un $39 \%$ en el número de plantas no injertadas e injertadas, respectivamente, necesarias para el cultivo de la misma superficie. De aquí surge la necesidad de estudios posteriores de evaluación económica de tal práctica.

\section{AGRADECIMIENTOS}

Los autores agradecen a la empresa Dunia Export S.A. las facilidades dadas para la realización de este trabajo. 


\section{LITERATURA CITADA}

BERTIN, N.; GARY, C. Tomato fruit set and competition for assimilates, during the early production period. Acta Horiculturae., n.303 p.121-126, 1992.

COCKSHULL, K.E.; HO, L.C. Regulation of tomato fruit size by plant density and truss thinning. Journal of Horticultural Science, v.70, n.3, p.395-407, 1995.

COOPER, A.J. Rapid crop turn-round is possible with experimental nutrient film technique. Grower, n.79, p.1048-1052, 1973.

GONZÁLEZ, J. El injerto en hortalizas. In: VILARNAU, A.; GONZÁLEZ, J. Planteles: semilleros, viveros. Reus: Ediciones de Horticultura, 1999. p.121-128.
NAWASHIRO, T. Grafting of watermelon. Tsukuba: Tsukuba International Agricultural Training Centre (JICA), 1994. 12 p. (Apostila, Vegetable Crops Production Course).

NEDERHOFF, E.M.; DE KONING, A.N.M.; RIJSDIJK, A.A. Leaf deformation and fruit production of glasshouse grown tomato (Lycopersicon esculentum Mill.) as affected by $\mathrm{CO}_{2}$, plant density and pruning. Journal of Horticultural Science, v.67, n.3, p.411-420. 1992. PAPADOPOULOS, A.P.; ORMROD, D.P. Plant spacing effects on yield of the greenhouse tomato. Canadian Journal of Plant Science, n.70, p.297304, 1990.

PAPADOPOULOS,

A.P.;

PARARAJASINGHAM, S. The influence of plan spacing on light interception and use in greenhouse tomato (Lycopersicon esculentum Mill.): A review. Scientia Horticulturae, n.69, p.1-29, 1997.
PEIL, R.M.N.; GÁLVEZ, J.L.; MARTIN, A. Cultivo de pepino con técnica de solución nutritiva recirculante. In: CONGRESO IBÉRICO SOBRE GESTIÓN Y PLANIFICACIÓN DE AGUAS (SIMPOSIUM: EL AGUA Y SUS USOS AGRARIOS), 1., 1998, Zaragoza. Actas.. Zaragoza: Universidad de Zaragoza, 1998, CD Rom, n.208, 9p.

SÁNCHEZ, I. Optimización de la densidad de plantación y del número de tallos en tomate cultivado con la técnica de la lámina de nutrientes (NFT). Almería: UAL/ Escuela politécnica Superior, 1999. 150 p. Proyecto de fin de carrera.

STANGHELLINI, C. Transpiration of greenhouse crops. An aid to climate management. Wageningen: Wageningen Agricultural University, 1987. 150 p. (Tese doutorado). 